\section{EFSUMB Young Investigator Award 2019}

The winner at EUROSON 2019 of the Young Investigator 3000 euro prize was Rogier $\mathrm{R}$ Wildeboer, The Netherlands for the abstract entitled

\section{D Multiparametric Ultrasound for Prostate Cancer Diagnosis}

Rogier R Wildeboer, Ruud JG van Sloun, Hessel Wijkstra, and Massimo Mischi

Department of Electrical Engineering, Eindhoven University of Technology, Eindhoven, The Netherlands

\section{Introduction}

Prostate Cancer (PCa) is the most frequently occurring type of non-skin cancer among American [1] and European men [2]. Due to the lack of reliable imaging, however, $\mathrm{PCa}$ diagnosis relies on systematic needle biopsy without targeting suspicious lesions-in spite of its associated complications and known risk of underdiagnosis and overtreatment [3]. In the United States, for example, an estimated 1300000 systematic biopsies are performed annually [4], even- tually leading to only 164690 PCa diagnoses in 2018 [1]. Hence, there is a high clinical demand for reliable PCa imaging allowing targeted biopsy.

In recent years, a lion's share of clinical attention has been directed towards multiparametric magnetic resonance imaging (MRI). Despite being more cost-effective, practical and much wider available, ultrasound (US) has not received the same attention. Moreover, a multiparametric US approach, combining promising US techniques such as shear-wave elastography (which visualizes the increased stiffness of malignant tissue) and contrast-enhanced ultrasound (CEUS, which allows the characterization of angiogenic vasculature), has never been investigated. My objective was therefore to examine the full potential of US for PCa diagnosis.

\section{Methods}

To this end, I focussed on two challenges. First of all, the development of methods to analyse US videos in three dimensions (3D). A 3 D US implementation does not only allow rapid characterization of the entire prostate in a single recording, but it also enables the development of $3 \mathrm{D}$ features that are impossible to estimate in $2 \mathrm{D}$. The second challenge was to optimally combine different US techniques in a multiparametric fashion, improving on the diagnostic performance of US by exploiting the complementary information among its features.

\section{Results}

Both challenges required additional development of registration and segmentation tools to enable $3 \mathrm{D}$ histology-ultrasound fusion for accurate validation and data labelling. I showed how digital $3 \mathrm{D}$ reconstruction of the histology ensured a near-millimetre-scale matching [5]. Furthermore, a dedicated neural network architecture was designed for automated deep-learning-based (zonal) segmentation. This algorithm was successfully validated in three large datasets of 181 patients in total, acquired in several expert centres with different US scanners to ensure its robustness and generalizability, reaching an accuracy of over $97 \%$ [6].

Exploiting the $3 \mathrm{D}$ nature of $3 \mathrm{D}$ CEUS data, I developed a method to solve the full con-

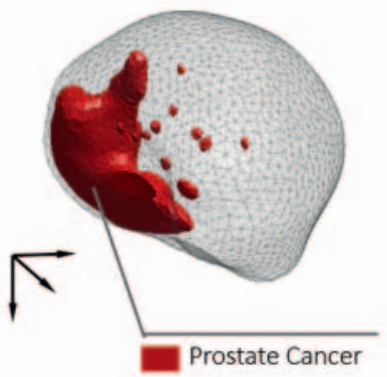

Novel Three-Dimensional Features

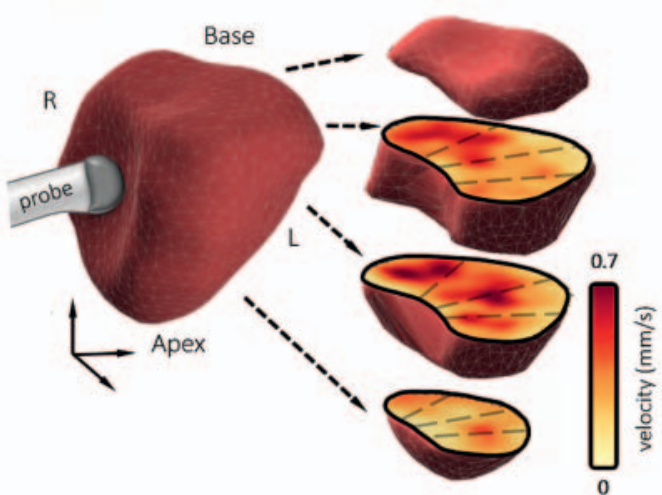

Multiparametric Combination
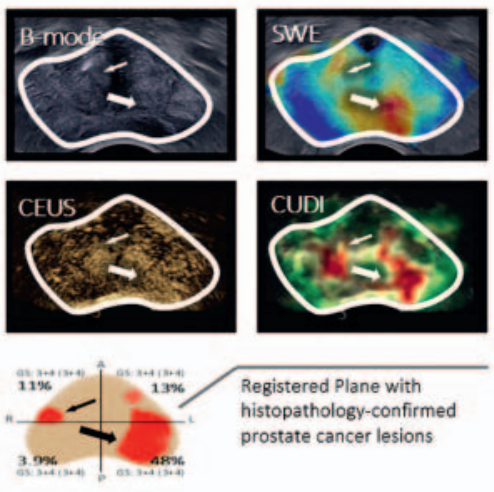
vective-dispersion equation describing the spreading of a contrast-bolus through the prostate [7]. This allowed the extraction of novel $3 \mathrm{D}$ features to characterize convection and dispersion in the (micro)vasculature. Using the $3 \mathrm{D}$ reconstructions of histopathology, these features were shown to localize PCa with a ROC curve-area of 0.80 in a preliminary set of 6 patients that underwent CEUS at the Second Affiliated Hospital of Zhejiang University (SAHZU, Hangzhou, China). Expanding on these results, now a range of $3 \mathrm{D}$ CEUS features can be extracted, from point-to-point dispersion to fractal dimensionality [8]. These features are conveniently visualized through a probabilistic tractography approach revealing the level of dispersion and convection in the $3 \mathrm{D}$ vascular network to the clinician [9].

Meanwhile, to investigate the feasibility of multiparametric US, machine learning by means of a Gaussian mixture model using only CEUS features already achieved a higher detection performance than individual parameters, increasing the accuracy from $73 \%$ to $81 \%$ [10]. Thus, in collaboration with clinical partners at the Academic Medical Centre (University of Amsterdam, Amsterdam, The Netherlands) and the Martini Klinik in Hamburg (University Hospital, Hamburg-Eppendorf, Germany), I conducted the first clinical study combining B-mode US, CEUS, its quantification, as well as shear-wave elastography [11]. A total of 50 patients referred for radical prostatectomy were enrolled in this study; my preliminary work on registration allowed me to accurately match the histopathology to the imaging planes and automatically segment the prostate. The first results show that clinical reviewers of mpUS can detect Gleason > 3 + 4 PCa with a sensitivity of $86 \%$, more than $10 \%$ higher than individual US techniques, without compromising the sensitivity [12]. Moreover, a computeraided detection algorithm, which was developed concurrently, was able to locate significant tumour regions with an ROCcurve area of 0.9 by actively exploiting the automatic zonal segmentation as well as a range of feature radiomics.
Also the first step to a complete $3 \mathrm{D}$ multiparametric analysis has been made. In a systematic-biopsy-validated 43-patient cohort that underwent $3 \mathrm{D}$ CEUS at the SAHZU, I showed that a multiparametric Gaussianmixture-model-based approach, including all the novel-developed 3D CEUS features, can improve over single-parametric investigation [8].

\section{Conclusion}

In my research, a $3 \mathrm{D}$ and multiparametric approach to US imaging of PCa was shown to be feasible and promising. Expanding on this work, a full $3 \mathrm{D}$ multiparametric US framework will be developed. Such a framework can be expected to enable costeffective biopsy targeting in the future.

\section{References}

[1] Siegel RL, Miller KD, Ahmedin ]. CA Cancer J Clin. 2018; 68(1): 7-30

[2] Ferlay J, Colombet M, Soerjomataram I, et al. Eur J Cancer. 2018; 103: 356-387

[3] Ukimura O, Coleman JA, de la Taille A, et al. Eur Urol. 2013; 63(2): 214-230

[4] Hutchinson RC, Costa DN, Lotan Y. Urol Oncol Semin Orig Investig. 2016; 34(7): 296-302

[5] Wildeboer RR, Schalk SG, Demi L, Wijkstra H, Mischi M. Biomed Phys Eng Express. 2017; 3(3): 35014

[6] van Sloun RJG, Wildeboer RR, Postema AW, et al. 2018 IEEE Int Ultrason Symp Proc. 2018

[7] Wildeboer RR, van Sloun RJG, Schalk SG, et al. IEEE Trans Med Imaging. 2018; 37(12): 2593-2602

[8] Wildeboer RR, van Sloun RJG, Huang P, Wijkstra H, Mischi M. 2018 IEEE Int Ultrason Symp Proc. 2018

[9] Wildeboer RR, Sloun RJG Van, Mannaerts CK, et al. 2018 IEEE Int Ultrason Symp Proc

[10] Wildeboer RR, Postema AW, Demi L, Kuenen MPJ, Wijkstra H, Mischi M. Eur Radiol. 2017; 27: 3226-3234

[11] Mannaerts CK, Wildeboer RR, Postema AW, et al. BMC Urol. 2018; 18(1): 98

[12] Mannaerts CK, Wildeboer RR, Van Kollenburg RAA, et al. Eur Urol Suppl. 2019; 18(1): e1884-e1885

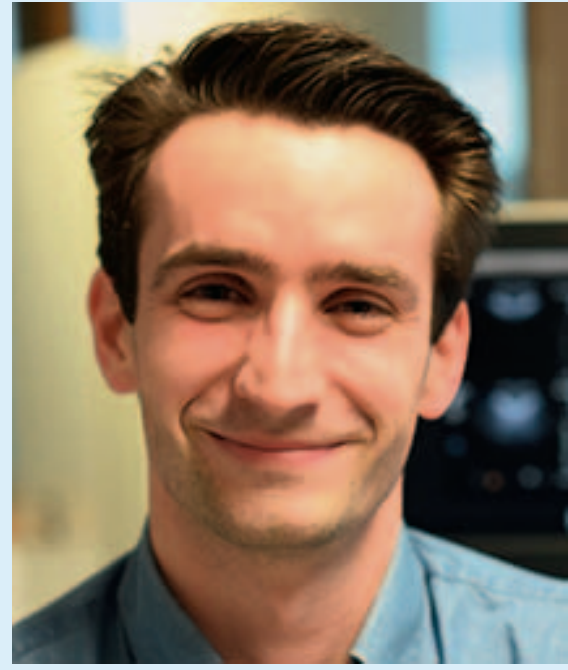

Rogier R. Wildeboer

Rogier R. Wildeboer received his B.Sc. degree with honours in Biomedical Technology and obtained an M.Sc. degree cum laude in both Biomedical Engineering and Applied Physics at the University of Twente. During his M.Sc. curriculum, he spend four months at the University College London to study the characterisation of magnetic nanoparticles for breast cancer treatment. His theses on photoacoustic breast cancer imaging as well as differential magnetometry for sentinel lymph node mapping were awarded several student research prizes. Currently, he is a PhD candidate at the Eindhoven University of Technology in collaboration with Philips Research and the Jeroen Bosch Hospital in 's-Hertogenbosch. Working closely together also with the Amsterdam University Medical Centres and the Martini Clinic in Hamburg, Germany, he studies the use of multiparametric and multidimensional ultrasound imaging of prostate cancer. Rogier authored more than 15 journal publications, three book chapters, and over 25 conference contributions, focusing on machine learning, biomedical imaging, and quantitative biophysical modelling. 